\title{
Evaluación de la inmunogenicidad de una vacuna recombinante antivirus hepatitis $B$ en mujeres adultas sanas, Santafé de Bogotá, D.C., julio 1997 - noviembre 1998
}

\author{
María T. Herrera Mendoza ${ }^{1}$, Jaime Escobar López², Clara I. Saavedra Abril ${ }^{3}$, Claudia P. Robles Roa ${ }^{4}$, \\ Fritz Gempeler ${ }^{5}$, Eduardo Egea Bermejo ${ }^{6}$, Antonio Iglesias Gamarra ${ }^{7}$
}

\begin{abstract}
Resumen
Este estudio se realizó con el propósito de evaluar la inmunogenicidad de la vacuna recombinante antivirus de la hepatitis B HEPRECOMB BERNA® y el desarrollo de efectos secundarios atribuibles a la vacuna y a la vía de aplicación, empleando y comparando dos dosis distintas en individuos sanos sin evidencia clínica de enfermedad hepática y sin evidencia serológica de contacto previo con VHB (virus hepatitis B). Igualmente se monitorizó la respuesta humoral in vivo inducida por la aplicación de esta vacuna. Se incluyeron 50 voluntarios del sexo femenino en Santafé de Bogotá, los cuales fueron divididos al azar en dos grupos:
\end{abstract}

El grupo 1, vacunado con tres dosis de $20 \mu \mathrm{g}$, y el grupo 2, vacunado con tres dosis de $10 \mu \mathrm{g}$; en ambos grupos se utilizó el esquema 0, 1, 6 meses y fueron observados durante 7 meses.

Los criterios de inclusión en el estudio fueron la participación voluntaria, edad mayor de 18 años y ausencia de antiHBsAg.

El título de anticuerpos fue significativamente mayor para el grupo 2 ( $\mathrm{P}=0.03$ ) a los dos y seis meses, aunque al séptimo mes los títulos se igualaron.

El trabajo concluyó que la vacuna recombinante antivirus de la hepatitis B HEPRECOMB BERNA $®$ con la dosis de $10 \mu \mathrm{g}$ tiene una mayor respuesta a los dos y seis meses de observación, que con la dosis de $20 \mu \mathrm{g}$, llegando a resultados similares al séptimo mes. Se recomienda realizar estudios con una muestra mayor.

Palabras clave: hepatitis B, vacuna recombinante antihepatitis B, inmunogenicidad.

\footnotetext{
${ }^{1}$ Servicio de Salud, Universidad Colegio Mayor de Cundinamarca, Santafé de Bogotá, D.C., Colombia

${ }^{2}$ Facultadad de Bacteriología, Universidad Colegio Mayor de Cundinamarca, Santafé de Bogotá, D.C., Colombia

${ }^{3}$ Servicio de Salud, Universidad Colegio Mayor de Cundinamarca, Santafé de Bogotá, D.C., Colombia

${ }^{4}$ Servicio de Salud, Universidad Colegio Mayor de Cundinamarca, Santafé de Bogotá, D.C., Colombia

${ }^{5}$ Laboratorio Walter Rothlisberger \& Co. Ltda., Santafé de Bogotá, D.C., Colombia

${ }^{6}$ Laboratorio de Biología Molecular e Inmunología. División Ciencias de la Salud. Universidad del Norte, Barranquilla, Colombia.

${ }^{7}$ Facultad de Medicina, Universidad Nacional de Colombia, Santafé de Bogotá, D.C., Colombia
} 


\title{
Evaluation of the immunogenicity of a hepatitis $B$ recombinant vaccine as used on healthy adult women
}

\begin{abstract}
A hepatitis $B$ antivirus pilot study was carried out to evaluate HEPRECOMB BERNA $®$ recombinant vaccine immunogenicity and the adverse effects which can be attributed to the vaccine and its application by using and comparing two different doses administered to healthy individuals who presented no clinical evidence of hepatic disease, no serological evidence of hepatic disease and no serological evidence of previous contact with HBV (Hepatitis B virus). The humoral in vivo response induced by the application of the vaccine was also monitored; 49 female volunteers from Santafé de Bogotá were included in the study and randomly divided into two groups.
\end{abstract}

Group 1 was vaccinated with three $20 \mu \mathrm{g}$ doses, whilst group 2 received three $10 \mu \mathrm{g}$ doses. In both groups the 0, 1 and 6 month scheme was used and women were observed for a period of 16 months. The inclusion criteria were: voluntary participation, 18 years or older and absence of anti HBsAg.

Antibody titre was significantly greater in group 2 at $2^{\text {nd }}$ and $6^{\text {th }}$ month of observation even though the titres were even at the end of the $7^{\text {th }}$ and $16^{\text {th }}$ months.

The present study concludes that the hepatitis $B$ antivirus recombinant vaccine HEPRECOMB BERNA $\otimes$ given in $10 \mu \mathrm{g}$ doses has a greater response in the first 2 months of observation than $20 \mu \mathrm{g}$ doses, similar results being found at the end of the seventh month. The recommendation is that studies should be done on bigger populations.

Key words: Hepatitis $B$, hepatitis $B$ antivirus recombinant vaccine, hepatitis $B$ vaccine immunogenicity, recombinant vaccine immunogenicity.

\section{Introducción}

La hepatitis $B$ es un problema de salud pública a nivel mundial. El virus se adquiere por inoculación parenteral o contacto sexual. Causa un cuadro agudo o crónico de enfermedad hepática (1); la infección crónica se asocia con el desarrollo de cirrosis hepática o carcinoma hepatocelular $(1,2,3)$.

La inmunización activa que emplea vacunas hemoderivadas o recombinantes constituye la única medida eficaz de prevención y control de la enfermedad (4). La hepatitis B puede infectar individuos de todas las edades y grupos étnicos. Son grupos de alto riesgo las personas sexualmente promiscuas, los drogadictos IV, y quienes tienen actividades laborales específicas (trabajadores de la salud).

La vacunación antihepatitis $B$ debe llevarse a cabo de acuerdo con las recomendaciones de la Organización Mundial de la Salud (5).
En nuestro país, las vacunas usadas en campañas masivas de vacunación, salvo excepciones aisladas (6), se basan en trabajos de investigación realizados en el país productor de la vacuna; sin embargo, es importante validar el empleo de las vacunas mediante estudios con población nativa que permitan definir la dosis óptima y el esquema adecuado a emplear, con el fin de inducir mediante la vacunación la tasa más alta de seroconversión y el índice más elevado de seroprotección y así lograr finalmente la prevención de la enfermedad.

En Colombia, las vacunas antihepatitis B de segunda generación obtenidas por tecnología recombinante han sido aplicadas desde 1986 y utilizadas en programas masivos de vacunación desde 1990. Tres vacunas diferentes se han comercializado y empleado, a saber: Heprecomb Berna $₫$, Heberbiovac $\mathrm{HB} \otimes$ y Engerix $\mathrm{B} \otimes$.

Los estudios realizados en nuestro país se han concentrado en el empleo y evaluación de las 
vacunas Herberbiovac $H B \otimes$ y Engerix $B \otimes$, demostrando su capacidad para inducir seroconversión y provocar seroprotección. Sin embargo, a pesar de ser empleada en programas de vacunación, y hasta donde es de conocimiento de los autores, no existían estudios que apoyaran el empleo de la vacuna Heprecomb de Berna® en el país y su uso se basa en trabajos realizados en Suiza, Japón o Bélgica, en los que se estudia el efecto de la vacuna en una población con características étnicas, genéticas, demográficas y epidemiológicas propias de las regiones de origen $(7,8,9,10)$. De acuerdo a recomendaciones de la casa fabricante, la vacuna Heprecomb® debe ser usada empleando el esquema 0,1 y 6 meses a dosis de $10 \mu \mathrm{g}$.

Puesto que un individuo puede adquirir la enfermedad antes, durante o después de la vacunación, resulta importante la respuesta inmune inmediata que sigue a la aplicación de la primera dosis y su comportamiento una vez aplicadas las dosis siguientes, con el fin de establecer cuándo y en qué medida el individuo vacunado presenta seroconversión y alcanza un estado de seroprotección. Así mismo es necesario establecer para nuestra población la existencia de individuos no respondedores, respondedores altos y respondedores bajos (6).

La evaluación de la vacuna, cuyo empleo se propone como medida eficaz para el control de la enfermedad, y el estudio de la respuesta humoral in vivo, inducida por su aplicación, brinda elementos experimentales inmediatos y objetivos para validar su empleo y permite definir su valor real como herramienta en el control y prevención de la enfermedad(11).

\section{Materiales y métodos}

El trabajo se diseñó como un estudio clínico experimental, prospectivo, comparativo, ciego simple de dos grupos homogéneos de mujeres entre 18 y 44 años de edad, cuya respuesta a la aplicación de la vacuna fue evaluada en forma independiente y comparada en el tiempo.

Todos los participantes fueron informados a través de consulta médica sobre los pormenores de la investigación, después de lo cual, manifestaron de forma escrita su consentimiento para participar. Se les realizó historia clínica, en la cual se consignaron antecedentes médicos familiares, datos de identificación, examen físico, edad, talla, peso, resultados de exámenes paraclínicos anteriores a la vacunación y registro de efectos adversos posteriores a la aplicación de cada una de las dosis.

Se evaluaron las siguientes variables:

\section{Variable dependiente}

Inmunogenicidad

La seroconversión se definió como la aparición, inducida por la aplicación de la vacuna, de anticuerpos IgG antiantígeno de superficie con una concentración $>1 \mathrm{mUl} / \mathrm{ml}$ en individuos seronegativos(12). La seroprotección se definió como la producción, inducida por la vacuna, de anticuerpo $\lg G$ antiantígeno de superficie con una concentración de $10 \mathrm{mUl} / \mathrm{ml}$ (4).

Los pacientes fueron clasificados como normorespondedores si su producción de antiHBsAg (anticuerpos antiantígeno de superficie de hepatitis B) estaba entre 10 y $100 \mathrm{mUl} / \mathrm{ml}$; hiporespondedores, entre $1-10 \mathrm{mUI} / \mathrm{ml}$, y no respondedores, aquéllos en quienes no se detectó ningún anticuerpo.

\section{Variable independiente \\ Criterios de inclusión}

Los sujetos involucrados cumplieron los siguientes requisitos: mujeres que manifestaron su deseo de participar voluntariamente, y con edades que oscilaron entre 18 y 44 años, con resultados negativos en las pruebas de detección de marcadores serológicos del VHB (antígeno de superficie, anticuerpos anti-antígeno central, anticuerpos anti-antígeno de superficie) y con valores normales de aminotransferasas (transaminasas basales). Después de satisfacer los criterios de selección se dividieron al azar en dos grupos de 25 individuos cada uno.

Dosis aplicada de la vacuna y esquema de vacunación:

Grupo 1: Esquema 0,1,6 meses a dosis de 20 $\mu \mathrm{g}$. Grupo 2: Esquema 0,1,6 meses a dosis de 10 $\mathrm{gg}$.

La vacunación fue realizada por personal médico y paramédico, el cual desconocía a qué grupo 
pertenecía cada individuo y la dosis aplicada, ya que recibía la vacuna lista para su aplicación de parte de los investigadores, quienes supervisaron permanentemente el procedimiento. Cada dosis de la vacuna se aplicó por vía intramuscular en la región deltoidea.

Cada grupo fue controlado así: una vez aplicada la vacuna se tomaron muestras de sangre venosa y se realizó la detección y cuantificación del anticuerpo IgG antígeno de superficie del VHB de acuerdo al siguiente plan de trabajo: se obtuvieron 5 muestras de cada paciente.

La duración del estudio fue de 16 meses y la observación de cada paciente fue de 7 meses, así: la muestra 1 se obtuvo dos días antes de la aplicación de la primera dosis; la muestra 2 se obtuvo un mes después de la primera dosis y antes de la aplicación de la segunda dosis; la muestra 3 fue obtenida un mes después de la segunda dosis; la muestra 4 fue obtenida 5 meses después de la segunda dosis; la muestra 5 fue obtenida un mes después de la tercera dosis.

Efectos adversos. Se evaluó la presencia de los siguientes efectos adversos secundarios a la aplicación de la vacuna Heprecomb® Berna: efectos locales como dolor, induración, eritema, inflamación, y efectos adversos de origen sistémico como cefalea, fiebre, malestar gastrointestinal, mareo y cansancio (13).

Las pruebas serológicas para detectar y cuantificar antiantígeno de superficie se obtuvieron de muestras de sangre venosa, $10 \mathrm{ml}$ en total, las cuales fueron centrifugadas a 3000 r.p.m. y el suero fue depositado en dos tubos de microcentrífuga de $1.5 \mathrm{ml}$ y conservados a $-20^{\circ} \mathrm{C}$ hasta el día en que se realizaron los ensayos. Estos fueron procesados empleando estuches de reactivos comerciales, de acuerdo con las instrucciones del fabricante en el IMX de ABBOTT ${ }^{\circledR}$ Laboratories $(14,15,16,17)$.

Las siguientes técnicas y estuches de reactivos fueron utilizados:

Detección de antígeno de superficie del VHB Técnica: inmunoensayo enzimático

Referencia: AUZYME ( ABBOTT Laboratories Diagnostics División)®
Detección y cuantificación de anticuerpos lgG anti-antígeno de superficie VHB

Técnica: inmunoensayo enzimático

Referencia:

1. AUSAB (ABBOTT Laboratories - Diagnostics División) $\mathbb{R}$

2. AUSAB Panel de cuantificación (ABBOTT Laboratories - Diagnostics División)®

Detección de anticuerpos anti-antígeno central del VHB

Técnica: inmunoensayo enzimático

Referencia

1. CORZYME (ABBOTT Laboratories - Diagnostics División)®

Métodos estadísticos. Los resultados fueron procesados en computador con el programa estadístico SPSS y utilizando las pruebas estadísticas Mann-Whitney Test para distribución libre, análisis de varianza para variables no paramétricas y chi cuadrado para proporciones; se hallaron también los percentiles 5,50 y 95 . Se consideró significativo $\mathrm{P} \leq 0.05$.

\section{Resultados}

Se incluyeron 50 mujeres voluntarias sanas, de las cuales terminaron el estudio 49, pues se retiró un individuo del grupo 1 por falta de colaboración. El promedio de edad fue de 21.2 años para el grupo 1 y de 22.2 con percentil 5 de 19 años y percentil 95 de 28 años para el grupo 2, sin diferencia estadísticamente significativa entre los dos grupos (cuadro 1).

Los valores basales del antiantígeno de superficie fueron negativos en los dos grupos (cuadros 2y3).

Un mes después de la aplicación de la primera dosis, los valores obtenidos en los dos grupos no presentaron una diferencia estadísticamente significativa. Un mes después de la segunda dosis, los valores para el grupo 1 fueron de $163 \pm 171 \mathrm{mUl} / \mathrm{ml}$ y para el grupo 2 de $312 \pm 280$ $\mathrm{mUl} / \mathrm{ml}$, con una diferencia estadísticamente significativa $(p=0.03)$. A los 6 meses, los valores para el grupo 1 fueron de $339 \pm 345 \mathrm{mUl} / \mathrm{ml}$ y para el grupo 2 de $603 \pm 535 \mathrm{mUl} / \mathrm{ml}$, con una diferencia significativa de $(p=0.01)$. Los valores observados un mes después de la aplicación de la última dosis (7 meses) fueron de $985 \pm 69 \mathrm{mUl} / \mathrm{ml}$ para el grupo 
Cuadro 1. Valores promedio de variables en estudio según grupos.

\begin{tabular}{lrrrrr}
\hline & \multicolumn{2}{c}{ GRUPO 1 } & \multicolumn{3}{c}{ GRUPO 2 } \\
\hline \hline & \multicolumn{1}{c}{$X$} & S & \multicolumn{1}{c}{$X$} & SD & P \\
\hline \hline EDAD (ANNOS) & 21.2 & 5 & 22.2 & 6 & NS \\
AST & 17.4 & 4.5 & 28.1 & 7.3 & 0.001 \\
ALT & 12.7 & 4 & 18.4 & 5.9 & 0.003 \\
& & & & & \\
ANTI-HBs (mU/ml) & Negativo & & Negativo & & \\
Basal & 7.7 & 10.7 & 10.1 & 10.9 & NS \\
1 Mes & 163 & 171 & 312 & 280 & 0.03 \\
2 meses & 339 & 345 & 603 & 535 & 0.01 \\
6 meses & 985 & 69 & 984 & 79 & NS \\
7 meses & & & & &
\end{tabular}

Cuadro 2. Estudios paraclínicos previos en evaluación vacuna antihepatitis $\mathrm{B}$ Heprecomb Berna.

\begin{tabular}{|c|c|c|c|c|c|c|c|c|}
\hline \multicolumn{9}{|c|}{ GRUPO I - DOSIS 20 ug } \\
\hline $\begin{array}{l}\text { AST/ } \\
\text { GOT }\end{array}$ & $\begin{array}{l}\mathrm{ALT} / \\
\mathrm{GPT}\end{array}$ & $\begin{array}{c}\mathrm{Hbs} A \\
\mathrm{~g}\end{array}$ & $\begin{array}{l}\text { anti- } \\
\mathrm{HBC}\end{array}$ & & & -HBs $n$ & nUliml & \\
\hline $\mathrm{U} / \mathrm{L}$ & $\mathrm{U} / \mathrm{L}$ & & & M1 & M2 & M3 & M4 & M5 \\
\hline & & & & & & & & \\
\hline 13 & 6 & $\mathrm{Neg}$ & Neg & 0.0 & 1.5 & 88.9 & 60.8 & $>1000.0$ \\
\hline 22 & 16 & Neg & $\mathrm{Neg}$ & 0.0 & 3.8 & 25.1 & 183.6 & $>1000.0$ \\
\hline 29 & 27 & $\mathrm{Neg}$ & Neg & 0.0 & 4.3 & 178.0 & 54.0 & $>1000.0$ \\
\hline 12 & 11 & $\mathrm{Neg}$ & Neg & 0.0 & 8.2 & 728.0 & $>1000.0$ & $>1000.0$ \\
\hline 17 & 13 & Neg & Neg & 0.0 & 0.0 & 48.2 & 103.4 & $>1000.0$ \\
\hline 19 & 15 & Neg & Neg & 0.0 & 2.5 & 108.2 & 181.2 & $>1000.0$ \\
\hline 21 & 15 & Neg & $\mathrm{Neg}$ & 0.0 & 3.7 & 221.9 & 413.4 & $>1000.0$ \\
\hline 26 & 16 & Neg & Neg & 0.0 & 8.2 & 62.6 & $>1000.0$ & $>1000.0$ \\
\hline 10 & 10 & Neg & Neg & 0.0 & 0.0 & 164.1 & 221.4 & $>1000.0$ \\
\hline 17 & 15 & Neg & Neg & 0.0 & 37.9 & 141.1 & $>1000.0$ & $>1000.0$ \\
\hline 16 & 12 & $\mathrm{Neg}$ & $\mathrm{Neg}$ & 0.0 & 0.0 & 221.1 & 533.6 & $>1000.0$ \\
\hline 15 & 17 & Neg & Neg & 0.0 & 18.3 & 2.7 & 96.2 & 657.3 \\
\hline 17 & 12 & $\mathrm{Neg}$ & $\mathrm{Neg}$ & 0.0 & 0.0 & 122.8 & 802.1 & $>1000.0$ \\
\hline 14 & 9 & $\mathrm{Neg}$ & $\mathrm{Neg}$ & 0.0 & 19.5 & 96.0 & 120.6 & $>1000.0$ \\
\hline 17 & 14 & $\mathrm{Neg}$ & $\mathrm{Neg}$ & 0.0 & 0.0 & 5.9 & 160.5 & $>1000.0$ \\
\hline 19 & 11 & $\mathrm{Neg}$ & Neg & 0.0 & 31.9 & 423.7 & 176.3 & $>1000.0$ \\
\hline 19 & 11 & Neg & Neg & 0.0 & 0.0 & 118.9 & 139.4 & $>1000.0$ \\
\hline 13 & 10 & $\mathrm{Neg}$ & $\mathrm{Neg}$ & 0.0 & 15.3 & 98.6 & 296.6 & $>1000.0$ \\
\hline 15 & 14 & Neg & Neg & 0.0 & 16.9 & 524.6 & 127.2 & $>1000.0$ \\
\hline 18 & 10 & $\mathrm{Neg}$ & $\mathrm{Neg}$ & 0.0 & 2.8 & 98.6 & $>1000,0$ & $>1000.0$ \\
\hline 12 & 10 & $\mathrm{Neg}$ & $\mathrm{Neg}$ & 0.0 & 2.9 & 121.2 & 142.7 & $>1000.0$ \\
\hline 18 & 11 & $\mathrm{Neg}$ & $\mathrm{Neg}$ & 0.0 & 0.0 & 70.2 & 91.8 & $>1000.0$ \\
\hline 24 & 11 & $\mathrm{Neg}$ & $\mathrm{Neg}$ & 0.0 & 0.0 & 235.1 & 243.1 & $>1000.0$ \\
\hline 15 & 10 & $\mathrm{Neg}$ & $\mathrm{Neg}$ & 0.0 & $\mathrm{NR}$ & 26.1 & 8.0 & $>1000.0$ \\
\hline
\end{tabular}

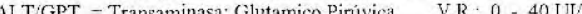

1 y para el grupo 2 de $984 \pm 79 \mathrm{mUl} / \mathrm{ml}$, sin diferencia significativa $(p=N S)$ (cuadro 1).

En la figura 1 se observa la dispersión de los valores obtenidos en los dos grupos bajo estudio, en los cuales, 1 mes después de haber aplicado la segunda dosis, dos individuos del primer grupo y uno del segundo aún no habían adquirido seroprotección. La aplicación de $20 \mu \mathrm{g}$ en un individuo del primer grupo obtuvo un valor de 8 $\mathrm{mUl} / \mathrm{ml}$ al sexto mes, pero al final alcanzó un valor de $1.000 \mathrm{mUl} / \mathrm{ml}$.

La presencia de efectos secundarios locales, evaluando la vía de aplicación en el grupo 1 (cuadro 4 ), fue de $41.7 \%$ y para el grupo 2 de
$24 \%$, sin diferencia estadísticamente significativa entre los grupos. Al evaluar las reacciones sistémicas se encontró que el $58.3 \%$ de los individuos presentó al menos un efecto secundario atribuible a la medicación en el grupo 1 , mientras que en el grupo 2 la ausencia de este tipo de efectos fue del $68 \%$. Dentro de los efectos secundarios locales más frecuentes reportados, se encontró el dolor (G1: $45.8 \%$ y G2 :52\%), ardor (G1:33.3\% y G2:20\%), el prurito, la induración (G1:45.8\% y G2:52\%) y tumefacción en menores proporciones (cuadro 4). En cuanto a reacciones

Cuadro 3. Estudios paraclínicos previos en evaluación vacuna antihepatitis $B$ Heprecomb Berna.

\begin{tabular}{|c|c|c|c|c|c|c|c|c|}
\hline \multicolumn{9}{|c|}{ GRUPO II-DOSIS 10 ug } \\
\hline \multirow{2}{*}{$\begin{array}{c}\text { AST/GO } \\
T \\
U / L\end{array}$} & \multirow{2}{*}{\begin{tabular}{|c|} 
ALT/GP \\
$T$ \\
$U / L$ \\
\end{tabular}} & \multirow{2}{*}{$\begin{array}{c}\mathrm{HbsA} \\
\mathrm{g} \\
\end{array}$} & \multirow{2}{*}{$\begin{array}{l}\text { anti- } \\
\mathrm{HBC}\end{array}$} & \multicolumn{5}{|c|}{ anti-HBs $\mathrm{mUl} / \mathrm{ml}$} \\
\hline & & & & M1 & $M 2$ & M3 & M4 & M5 \\
\hline 30 & 16 & $\mathrm{Neg}$ & $\mathrm{Neg}$ & 0,0 & 4,6 & 45,7 & 165,8 & $>1000.0$ \\
\hline 25 & 18 & Neg & Neg & 0.0 & 3,7 & 86,0 & 707,3 & $>1000.0$ \\
\hline 30 & 23 & Neg & Neg & 0,0 & 20,2 & 830,5 & 895,1 & $>1000.0$ \\
\hline 26 & 15 & Neg & Neg & 0,0 & 16,8 & 626,9 & 603,9 & $>1000.0$ \\
\hline 27 & 16 & $\mathrm{Neg}$ & Neg & 0,0 & 5,18 & 268,4 & 72 & $>1000.0$ \\
\hline 36 & 29 & Neg & Neg & 0,0 & 18,6 & 609,0 & 161,5 & $>1000.0$ \\
\hline 41 & 31 & Neg & Neg & 0,0 & 12,4 & $<1000$ & 350,2 & $>1000.0$ \\
\hline 32 & 20 & $\mathrm{Neg}$ & Neg & 0,0 & 5,62 & 108,2 & 384 & 604,9 \\
\hline 26 & 14 & $\mathrm{Neg}$ & Neg & 0,0 & 4,35 & 181,4 & 1000 & $>1000.0$ \\
\hline 33 & 20 & $\mathrm{Neg}$ & $\mathrm{Neg}$ & 0,0 & 8,76 & 226,3 & 1000 & $>1000.0$ \\
\hline 20 & 15 & $\mathrm{Neg}$ & $\mathrm{Neg}$ & 0,0 & 16,0 & 381,0 & 489 & $>1000.0$ \\
\hline 30 & 23 & $\mathrm{Neg}$ & $\mathrm{Neg}$ & 0,0 & 7,2 & 198,8 & 1000 & $>1000.0$ \\
\hline 23 & 18 & $\mathrm{Neg}$ & $\mathrm{Neg}$ & 0,0 & 34,7 & 428,7 & 1000 & $>1000.0$ \\
\hline 29 & 15 & $\mathrm{Neg}$ & Neg & 0.0 & 6,70 & 65,8 & 1000 & $>1000,0$ \\
\hline 28 & 19 & $\mathrm{Neg}$ & $\mathrm{Neg}$ & 0.0 & 3,70 & 179,9 & 1000 & $>1000.0$ \\
\hline 24 & 17 & $\mathrm{Neg}$ & $\mathrm{Neg}$ & 0,0 & 11,2 & 5,8 & 46,8 & $>1000.0$ \\
\hline 53 & 31 & $\mathrm{Neg}$ & $\mathrm{Neg}$ & 0,0 & 2,93 & 359 & 384,9 & $>1000.0$ \\
\hline 20 & 15 & $\mathrm{Neg}$ & $\mathrm{Neg}$ & 0.0 & 3,99 & 155 & 202,9 & $>1000.0$ \\
\hline 26 & 17 & $\mathrm{Neg}$ & $\mathrm{Neg}$ & 0,0 & 0.0 & 210,5 & 691 & $>1000.0$ \\
\hline 25 & 16 & $\mathrm{Neg}$ & $\mathrm{Neg}$ & 0,0 & 0,0 & 71,9 & 257,6 & $>1000.0$ \\
\hline 25 & 16 & $\mathrm{Neg}$ & Neg & 0,0 & 0,0 & 71,7 & 968,4 & $>1000.0$ \\
\hline 26 & 14 & Neg & $\mathrm{Neg}$ & 0,0 & 44.7 & 454,7 & 929,9 & $>1000,0$ \\
\hline 26 & 25 & $\mathrm{Neg}$ & $\mathrm{Neg}$ & 0,0 & 19,2 & 733,4 & 1000 & $>1000.0$ \\
\hline 26 & 11 & Neg & Neg & 0,0 & 0,0 & 161,8 & 529,4 & $>1000.0$ \\
\hline 15 & 6 & Neg & Neg & 0.0 & 4,1 & 141,0 & 255 & $>1000.0$ \\
\hline
\end{tabular}

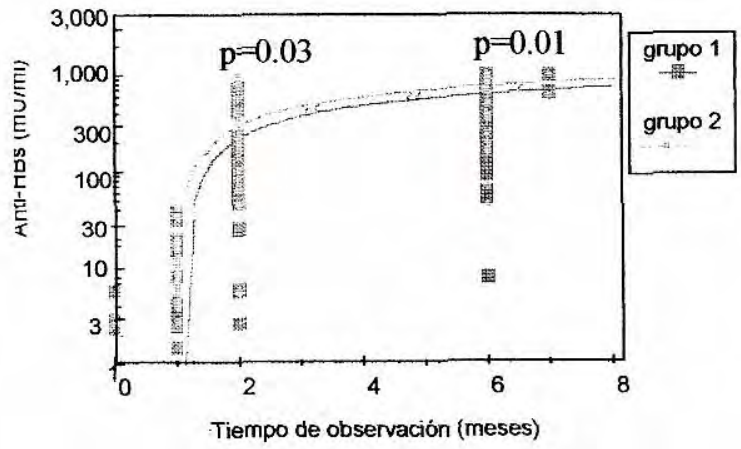

Figura 1. Comparación de antiHBs $(\mathrm{mU} / \mathrm{ml})$, dos esquemas de tratamiento. 
sistémicas, los efectos más frecuentes fueron las nauseas con diferencia significativa $(p=0.03)$, mareo (G1:20.8 \% y G2:8.0 \%), cefalea (G1:20.8\% y $\mathrm{G} 2: 8.0 \%$ ), nauseas (G1:16.7\% y $\mathrm{G} 2: 0.0 \%$ ), diarrea (G1:12.5\% y $\mathrm{G} 2: 0.0 \%$ ) (cuadro 4).

Cuadro 4. Distribución porcentual de presencia de efectos secundarios no inmunológicos según grupos en estudio.

\begin{tabular}{|c|c|c|c|c|c|}
\hline & \multicolumn{2}{|c|}{ GRUPO 1} & \multicolumn{2}{|c|}{ GRUPO 2} & \multirow[t]{2}{*}{$\mathrm{P}$} \\
\hline & $\mathrm{N}$ & $\%$ & $\mathrm{n}$ & $\%$ & \\
\hline TOTAL PACIENTES & 24 & 100 & 25 & 100 & \\
\hline \multicolumn{6}{|l|}{ REACCIONES LOCALES } \\
\hline Ninguna & 14 & 58,3 & 19 & 76 & NS \\
\hline Dolor & 11 & 45,8 & 13 & 52 & NS \\
\hline Ardor & 8 & 33,3 & 5 & 20 & NS \\
\hline Prurito & 3 & 12,5 & 1 & 4 & NS \\
\hline Induración & 3 & 12,5 & 0 & 0 & NS \\
\hline Eritema & 2 & 8,3 & 0 & 0 & NS \\
\hline Tumefacción & 1 & 4,2 & 0 & 0 & NS \\
\hline \multicolumn{6}{|l|}{ REACCIONES SISTEMICAS } \\
\hline Ninguna & 10 & 41,7 & 8 & 32 & NS \\
\hline Dolor en el brazo & 9 & 37,5 & 14 & 56 & NS \\
\hline Mareo & 5 & 20,8 & 1 & 4 & NS \\
\hline Cefalea & 5 & 20,8 & 2 & 8 & NS \\
\hline Nauseas* & 4 & 16,7 & 0 & 0 & 0.03 \\
\hline Diarrea & 3 & 12,5 & 0 & 0 & NS \\
\hline Fiebre & 2 & 8,3 & 1 & 4 & NS \\
\hline Fatiga & 1 & 4,2 & 1 & 4 & NS \\
\hline Erupción & 1 & 4,2 & 0 & 0 & NS \\
\hline Vómito & 0 & 0 & 0 & 0 & NS \\
\hline Artralgias & 0 & 0 & 0 & 0 & NS \\
\hline Somnolencia & 0 & 0 & 2 & 8 & NS \\
\hline
\end{tabular}

\section{Discusión}

En el estudio se compararon dos dosis de la vacuna Heprecomb ${ }^{\circledR}$ de Berna: $10 \mu \mathrm{g}$ y $20 \mu \mathrm{g}$, dosis recomendadas, respectivamente, por la casa fabricante y la OMS, para la inmunización contra la hepatitis $B$.

La metodología se estableció de acuerdo a recomendaciones de estudios previos que determinaron esquema de vacunación, vía de aplicación, condiciones de almacenamiento, etc. $(18,19)$. El esquema seleccionado fue $0,1,6$ meses (1), ya que se obtiene un nivel de seroprotección y seroconversión adecuado y rápido. La vía de aplicación de la vacuna fue intramuscular en la región deltoides $(20,21,22)$. Respecto a la conservación se mantuvo el almacenamiento de las vacunas de 2 a $8^{\circ} \mathrm{C}$.

Los dos grupos del presente estudio fueron conformados por mujeres jóvenes con un promedio de edad de 22 años, hecho que explica la excelente respuesta a la vacuna, lo que coincide con lo descrito por la literatura $(23,24)$. La explicación de por qué ellas responden mejor que los varones aún no ha sido determinada. En cuanto a la edad, se ha informado que la respuesta es menor hacia los 40 años $(25,26)$.

Este trabajo mostró que el grupo 2, en el cual se utilizó la dosis $10 \mu \mathrm{g}$, presentó mayores títulos de seroconversión y seroprotección un mes después de la aplicación de la primera y segunda dosis con respecto al grupo 1, pero al final de la inmunización ambos grupos alcanzaron títulos a $1000 \mathrm{mUl} / \mathrm{ml}$, excepto un individuo en cada grupo, con títulos de $657 \mathrm{mUl} / \mathrm{ml}$ y $604 \mathrm{mUl} / \mathrm{ml}$, respectivamente.

Teniendo en cuenta el resultado anterior, nos permitimos sugerir que la respuesta de anticuerpos es dosis dependiente en relación a las primeras inoculaciones.

En cuanto a los efectos adversos no inmunológicos, al disminuir la dosis disminuye el informe de éstos, especialmente nauseas; $\sin$ embargo, en ninguno de los dos grupos se informó de artralgias, exantemas, neuralgias o anafilaxis, lo que coincide con los trabajos realizados en Japón y Suiza (27) por la casa fabricante.

En el presente estudio no se encontraron sujetos no respondedores, lo que resulta similar a otros trabajos realizados en Colombia, en los que se afirma que los haplotipos descritos en EE.UU. y Japón para la no respuesta, especialmente el HLAB8, SCO1, DR3 (40, 47), HLAB W54-DR4DRW53-DQW4 $(28,29)$, no han sido hallados aquí. Sin embargo, los estudios sobre este tema aún no son suficientemente claros en el país.

Por lo anterior se puede concluir que tanto la dosis de $10 \mu \mathrm{g}$ como $20 \mu \mathrm{g}$ de la vacuna recombinante antihepatitis B Heprecomb $₫$ de Berna, permite a los individuos alcanzar, una vez terminado el esquema de vacunación, un nivel de seroprotección adecuado, lo que permite clasificarlos en la categoría de normorrespondedores. Sin embargo, en el grupo 1, un paciente se comportó como respondedor lento. Es importante el hecho de que el grupo 2, vacunado con la dosis de $10 \mu \mathrm{g}$, alcanzó más rápidamente el nivel de seroconversión y seroprotección que el grupo 1. El nivel de seroconversión alcanzado por el grupo 
2 fue mayor desde un mes después de la aplicación de la primera dosis, lo cual es relevante clínicamente ya que esto protege al paciente de contagio con el virus de la hepatitis $B$ desde este instante hasta finalizar su inmunización. De igual forma, el grupo 2, vacunado con la dosis de 10 ug, informó menos efectos secundarios no inmunológicos localizados y sistémicos que el grupo 1.

Recomendaciones. Por los resultados obtenidos, se recomienda reproducir este estudio en poblaciones mayores, que permitan confirmar que la dosis de $10 \mu \mathrm{g}$ es la apropiada para nuestro medio, con un estudio con diseño doble ciego, ya que esto implicaría menores costos para una país precario en recursos económicos y permitiría masificar las campañas de vacunación.

\section{Agradecimientos}

A Alicia Moyano Iregui, Rectora U.C.M.C., a Bertha Cecilia Díaz de Arana, Vicerrectora Académica, a Melba Nidia León y a la División de Investigaciones, UCMC; a los Laboratorios Walter Rothlisberger y Abbott Laboratories de Colombia; a Juan Carlos Rojas, Epidemiología y Administración Hospitalaria, a Janeth Navarrete, a Gladys Pinilla y a Clara López de Mesa.

\section{Referencias}

1. Scherlack S. The natural history of hepatitis B. Posgrad med J. 1987;6 (supp):7-11.

2. McLean AA, Guess HA, Scolnick EM. Sub optima reponse to hepatitis $B$ vaccine given by injection into the buttock. MMWR, 1985;34:105-108.

3. Bealsy P. The major etiology of hepato-celular carcinoma. Hepatitis virus cancer. 1998;61(10):1942-1956

4. Fields BN, Knipe DM, Chanock RM. Hepadnaviridae and the replication: In (eds). Fundamental Virology. Raven press $2^{\text {nd }}$ Edition. New York, 1991;989-999.

5. Centers for Disease Control. Protection against viral hepatitis MMWR, 1999;39 (5/2):15.

6. Diaz J, Ospina S, Zapata LD, Arroyave A, Marzo G. Respuesta a una vacuna contra hepatitis $B$ en trabajadores de la salud, Antioquia 1994. Boletín Epidemiologico de Antioquia, año XIX Octubre-Diciembre. 1994.

7. Andre FE. Owerview of a 5 year clinical experience with a yeast-derived hepatitis B vaccine. Vaccine 1990;8:574-578.

8. Just M, Berger R, Wegmann A et al. Induction of antiHBsAg antibodies with a new vaccine produced by genetic technology. Schweiz Rundschau Med 1998;51:1407-1409.
9. Hollinger F.B. Factors influencing the immune response to hepatitis $B$ vaccine, booster dose guidelines and vaccine protocol recommendations. American Journal of Medicine. 1989;87:(55 A)365.

10. Scolnick EM, McLean AA, West. DJ, McAleerWJ, Miller WS, Bynack EB. Clinical evaluation in healthy adults of a hepatitis $B$ vaccine made by recombinant DNA. JAMA 1984;251:2812-2815.

11. Hadler SC. Hepatitis B virus infection and health care workers. Vaccine 1990;8:5-24

12. Winter AP, Follett EAC, Mclntyre J, et al. Influence of smoking on inmunological responses to hepatitis $B$ vaccine. Vaccine 1994;12771-2.

13. Berna. Hepatitis viral: conceptos actuales y esquemas de control. Hepatitis 1997; Vol:1; 1:11-12.

14. Auszyme package insert. ABBOTT Laboratories Diagnostics Division. Chicago, IL, 1995

15. Corzyme package insert. ABBOTT Laboratories Diagnostics Division. Chicago, IL, 1995

16. Ausab package insert. ABBOTT Laboratories Diagnostics Division. Chicago, IL, 1995

17. Ausab quantification panel package insert. ABBOTT Laboratories Diagnostics Division. Chicago, IL, 1995

18. Schmidt M, Deinhardt FJ. Vaccination against hepatitis B: comparison of three different vaccination schedules. dilg Infect. Dis. 1989;160:776-9.

19. Hadler SC, de Monzón M, Lugo DR. Effect of timing of hepatitis $B$ vaccine doses on response to vaccine in Yuepa Indians. Vaccine 1989; 7:106-10.

20. Shaw FE jr, Guess HA, Roets JM et al. The effect of anatomic injection site, age and smoking on the immune response to hepatitis B vaccinacion. Vaccine 1989 (in press).

21. De Falla F, Rinaldi E, Santoro $D$ et al. Immune response to hepatitis $B$ vaccine given at different injection sites and by different routes: a controlled randomized study. $J$ epidemid 1988;4:256-258.

22. UkenaT, Esber H, Bessette R et al. Site of injection and response to hepatitis B vaccine. Engl J. Med. 1985;313:579580.

23. Seef LB, Koff RS. Passive and active immunoprofylaxis of hepatitis B. Gastroenterology 1984;86:958-081.

24. Just M, Berger R, Wegmann A et al. Inducción de anticuerpos HBs con una vacuna producida por tecnología genética. Schweiz Rundschau Med 1988;51:1407-1409.

25. Clements ML, Miskovsky E, Davidson M et al. Effect of age on the immunogenicity of yeast recombinant hepatitis $B$ vaccines containing surface antigen $(S)$ or pre $S 2+S$ antigens. J Infect Dis 1994;170:5 10-6.

26. Wood RC, MacDonald KL, Whites KE et al. Risk factors for lack of detectable antibody following hepatitis $B$ vaccinattion of Minnesota health care workers. JAMA 1993;270:2935-9 
27. Bryan JP, Craing PG, Reyes L, Hakre S, Jaramillo R, Harlan $\mathbf{H}$ et al. Randomized comparison of 5 and $10 \mu \mathrm{g}$ doses of two recombinant hepatitis $B$ vaccines. Vaccine 1995; Vol 13,11:978-982.

28. Watanabe $\mathrm{H}$, Matsushita S, Kamikawaji N et al. Immune suppression gene on HLA.Bk 54-DR4- DRw53 haplotype controls nonresponsiveness in humans to hepatitis $B$ sur- face antigen via CD8 suppressor T cell. Hum Inmunol 1988;2:9-17

29. Watanabe H, Okumura M, Hirayama K et al. HLA-BW54DR4-DRw53-DQw54 haplotype controls nonresponsiveness to hepatitis $B$ surface antigen via CD8-positive suppessorT cells. Tissue Antigens 1990;36:69-74. 